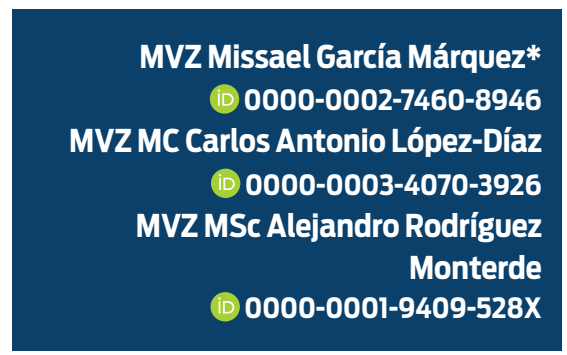

Universidad Nacional Autónoma de México.

* Autor para correspondencia: Correo electrónico: marquez.fmvz@gmail.com

\section{Casos clínicos referidos para terapia de ondas de choque en el hospital para équidos de la Facultad de Medicina Veterinaria y Zootecnia-UNAM (2011-2015)}

\section{Resumen}

Descripción de los casos. Se analizó una muestra de 232 pacientes remitidos para terapia de ondas de choque (TOCE), de 1292 casos clínicos que ingresaron al hospital para équidos de la Facultad de Medicina Veterinaria y Zootecnia de la UNAM durante 2011-2015. Se aplicaron en total 323 terapias; el $86.6 \%$ de los pacientes fueron prototipo warmblood con un promedio de edad de once años.

Hallazgos clínicos. La patología con mayor incidencia fue desmitis proximal del ligamento suspensor del menudillo o tercer ligamento interóseo, seguida de miositis dorsal y exostosis anillada.

Tratamiento y evolución. El tratamiento se llevó a cabo mediante terapia de onda de choque focal y terapia de onda de choque radial o terapia de presión radial.

Pruebas de laboratorio. Las pruebas de laboratorio no son necesarias porque TOCE no es una terapia invasiva.

Relevancia clínica. La TOCE representa el $17.9 \%$ del total de pacientes que ingresaron en el periodo de estudio. Se recibieron 46 pacientes por año en promedio, únicamente para TOCE. Se requeriría un estudio de casos clínicos totales del Hospital para Équidos para comparar las diferentes causas de remisión.

Palabras clave: ESWT, shock wave, terapia de ondas de choque, terapia física, equinos, TOCE.
Recibido: 2019-09-02 Aceptado: 2020-01-28 Publicado: 2020-05-13

Información y declaraciones adicionales en la página 10

(a) Derechos de autor: Missael García Márquez et al. 2020 acceso abierto $\boldsymbol{O}$

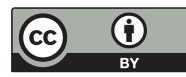

Una forma de citar este artículo:

García-Márquez M, López-Díaz CA, Rodríguez-Monterde A. Casos clínicos referidos para terapia de ondas de choque en el hospital para équidos de la Facultad de Medicina Veterinaria y Zootecnia-UNAM (2011-2015). Clínica veterinaria: abordaje diagnóstico y terapéutico. 2020;6:e54202061. doi:10.22201/fmvz.23958766e.202054 


\section{Clinical cases referred of shockwave therapy of equine hospital UNAM (2011-2015)}

\section{Abstract}

Case report. From 2011 to 2015, out of 1292 patients 232 we referend to the hospital for equine of the Departamento de Medicina Cirugía y Zootecnia de Équidos of the Facultad de Medicina Veterinaria y Zootecnia for shockwave therapy (ESWT) were analyzed. This therapy was applicated 323 in 232 patients, $86 \%$ of the patients are warmblood prototype and an average age of eleven years.

Clinical findings. The pathology with the highest incidence was proximal desmitis of the suspensory ligament or third interosseous ligament, followed by dorsal myositis and ringbone.

Treatment and evolution. The treatment was carried out by focal shock wave therapy and radial pressure therapy.

Laboratory test. The laboratory test is not necessary because the ESWT is not an invasive therapy.

Clinical relevance. The ESWT represents $17.9 \%$ of the total number of patients admitted in this study period. An average of 46 patients per year were received, for ESWT. A study of total clinical cases of the hospital for equine would be required to compare the causes of origin.

Keywords: SWT, Shock wave, Equine, ESWT, physical therapy. 


\section{Descripción de los casos}

Entre 2011 y 2015 se recibieron 1292 pacientes en el Hospital para Équidos (HE), los pacientes remitidos exclusivamente para TOCE representaron el $17.9 \%$ del total (Figura 1).

Durante el periodo de estudio se observó un incremento constante de casos clínicos remitidos al HE año con año, de 121 casos clínicos en 2011 a 352 en 2015; sin embargo, los pacientes remitidos por tOCE no incrementaron de la misma forma, quizá por el patrón cíclico de campeonatos nacionales e internacionales de algunas disciplinas ecuestres como salto y adiestramiento, que representaron el $92.67 \%$ del total de pacientes. En 2014, año en el que se celebraron los juegos centroamericanos en nuestro país, se remitieron 74 caballos para TOCE, cifra más alta en el periodo de estudio. Se requieren más años de estudio para la confirmación de esta hipótesis (Figura 2).

Se remitieron caballos de seis disciplinas ecuestres: adiestramiento (AD), carreras $(C A)$, resistencia o endurance $(E N)$, paseo $(P A)$, prueba completa $(P C)$, salto (SA). Los pacientes de salto representaron el $67.67 \%$ del total, los de adiestramiento el $25 \%$ y el $7.33 \%$ restante se divide en pacientes dedicados a paseo, resistencia, prueba completa y carreras. La cantidad de terapias que recibieron los pacientes por disciplina ecuestre se muestra en la Figura 3.

A pesar de que la raza del equino influye en la futura función zootécnica que realiza, esto no es una verdad absoluta, por ejemplo, se sabe que razas dentro del prototipo warmblood son preferidas por las disciplinas salto y adiestramiento, así como caballos cuarto de milla son populares en carreras, charrería y paseo; caballos españoles y frisones son utilizados en adiestramiento, baile, y el caballo pura sangre inglés es común en carreras, salto y adiestramiento. Debido a esto, la relación entre función zootécnica y raza o prototipo es muy dispersa y los resultados son meramente informativos. En este estudio, y con la muestra obtenida, el $86.6 \%$ de los pacientes fueron prototipo warmblood con un promedio de edad de once años.

\section{Revisión de registros}

Ya que es posible que un paciente reciba dos o más terapias durante una visita, sea de forma bilateral o en diferentes regiones anatómicas y con el objetivo de conocer la patología con mayor incidencia dentro de la muestra, se utilizó el número de terapias aplicadas para el análisis. La distribución completa se muestra en el Figura 4.

\section{Hallazgos clínicos e interpretación}

La desmitis proximal del suspensor fue la patología de mayor incidencia con $31 \%$ del total de terapias aplicadas. Todos caballos dedicados a las disciplinas ecuestres: salto, adiestramiento y prueba completa, de las que el $66 \%$ fueron lesiones en miembros torácicos y el $34 \%$ en miembros pélvicos. La relación se ejemplifica en la Figura 5.

La desmitis o inflamación del ligamento suspensor es una de las causas más comunes de claudicación en caballos, representa aproximadamente el 30 \% de 


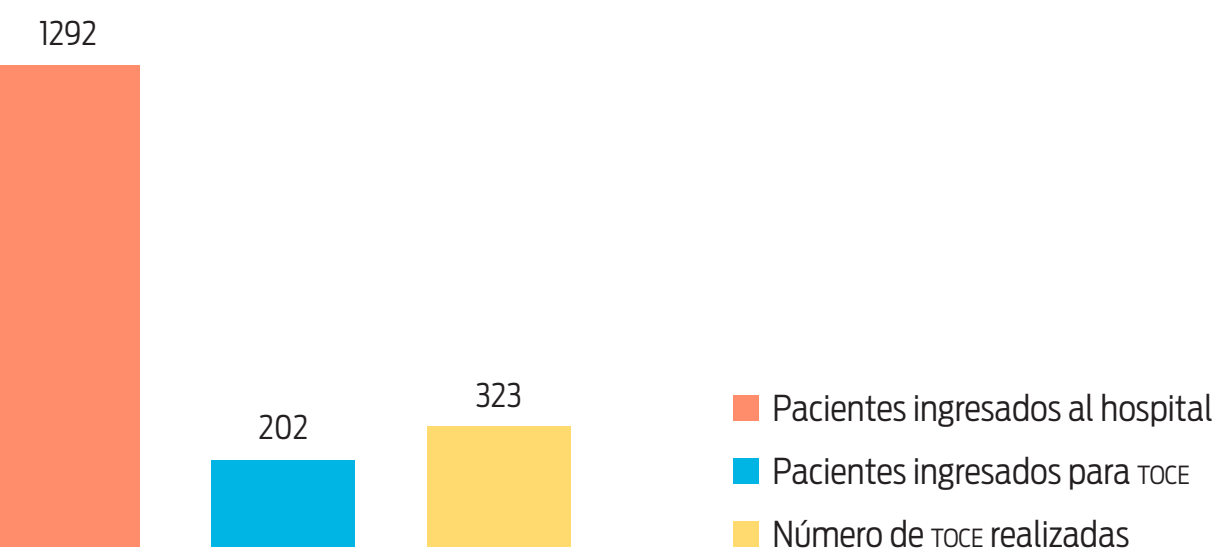

Figura 1. Pacientes recibidos entre 2011-2015.

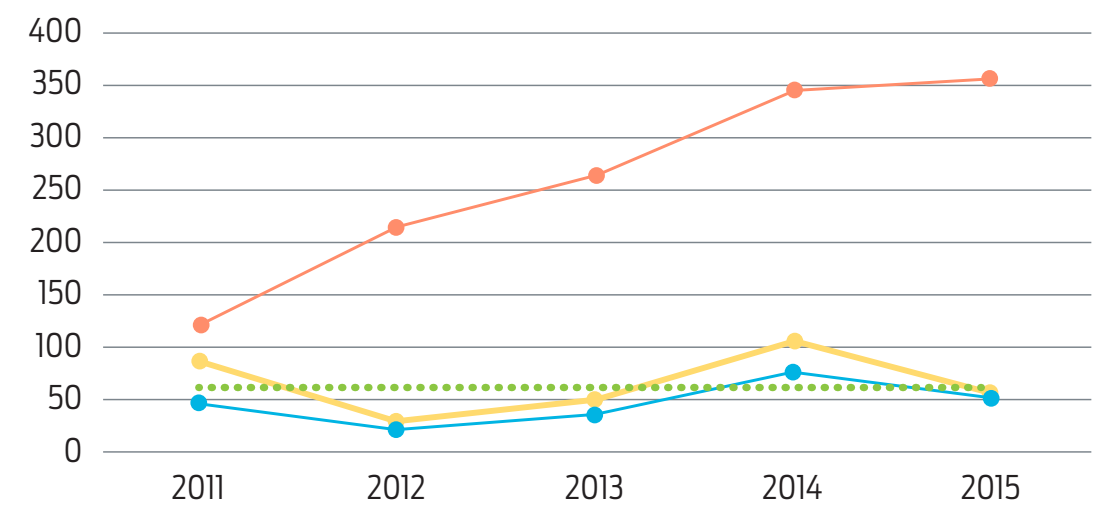

- - Pacientes ingresados al hospital

- Pacientes ingresados para toce

$\ldots$ Número de toce realizadas

..... Líneal (número de toce realizadas)

Figura 2. Tendencia en la cantidad de pacientes remitidos por TOCE.

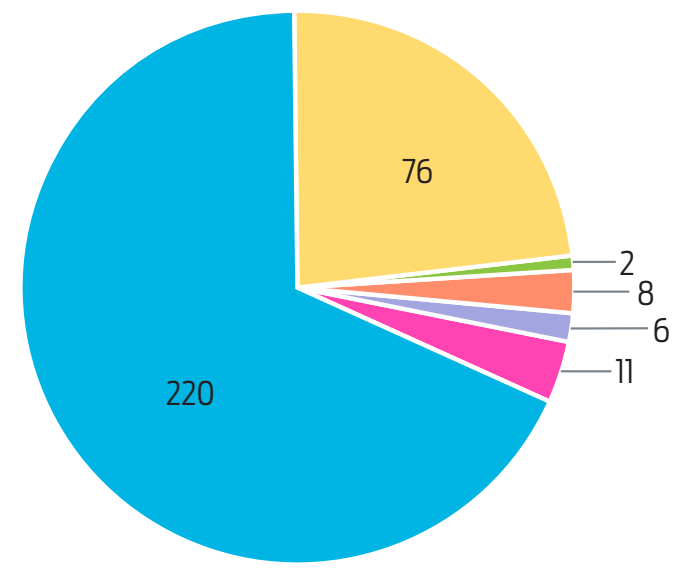

\section{$\mathrm{AD} \square \mathrm{CA} \square \mathrm{EN} \square \mathrm{PA} \square \mathrm{PC} \square \mathrm{SA}$}

Figura 3. Terapias aplicadas agrupadas por función zootécnica. 


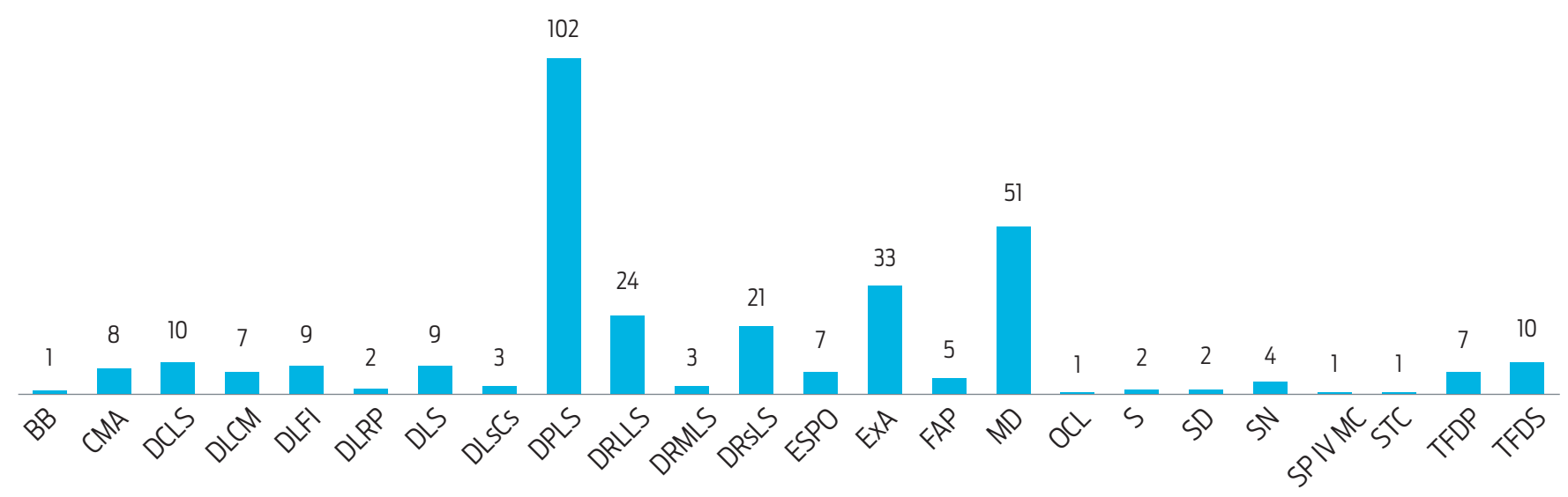

Figura 4. Patologías tratadas mediante TOCE en el Hospital para Équidos de la FMVZ-UNAM durante 2011-2015. BB bursitis bicipital, CMA osteocondroma, DCLS desmitis del cuerpo del suspensor, DLCM desmitis del colateral medial de la articulación interfalángica distal, DLFI desmitis de frenador inferior, DLRP desmitis del recto palmar, DLS desmitis del ligamento suspensor, DLsCs desmitis de los ligamentos colaterales, DPLS desmitis proximal del suspensor, DRLLS desmitis de la rama lateral del suspensor, DRMLS desmitis de la rama medial del ligamento suspensor, DRsLS desmitis de las ramas del suspensor, ESPO esparaván óseo, EXA exostosis anillada, FAP fractura de la apófisis piramidal o extensora, MD miositis dorsal, OCL osificación de los cartílagos alares, S sesamoiditis, SD sinovitis digital, SN síndrome navicular, SP IV MC exostosis del IV metacarpiano, STC síndrome del canal carpal, TDFP tendinitis del flexor digital profundo y TDFS tendinitis del flexor digital superficial.

\section{DPLS}

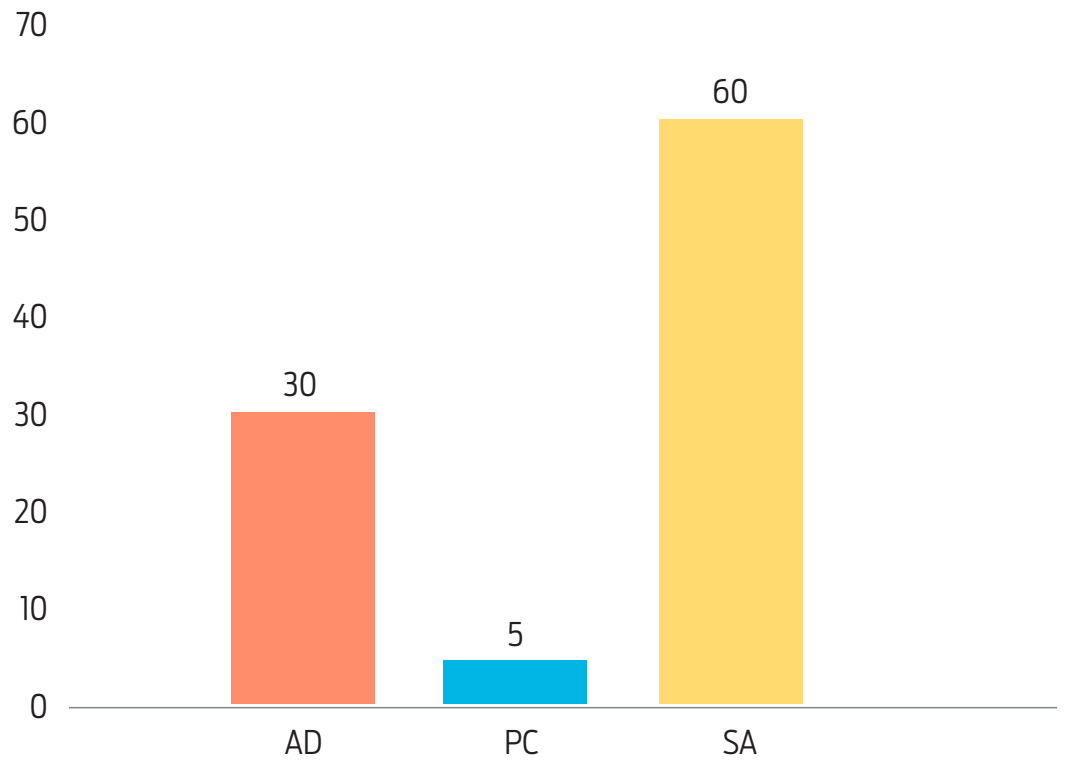

Figura 5. Prevalencia de desmitis proximal del ligamento suspensor (DPLS) en pacientes que recibieron tocE. DPLS desmitis proximal del ligamento suspensor, SA salto, PC prueba completa, AD adiestramiento. 


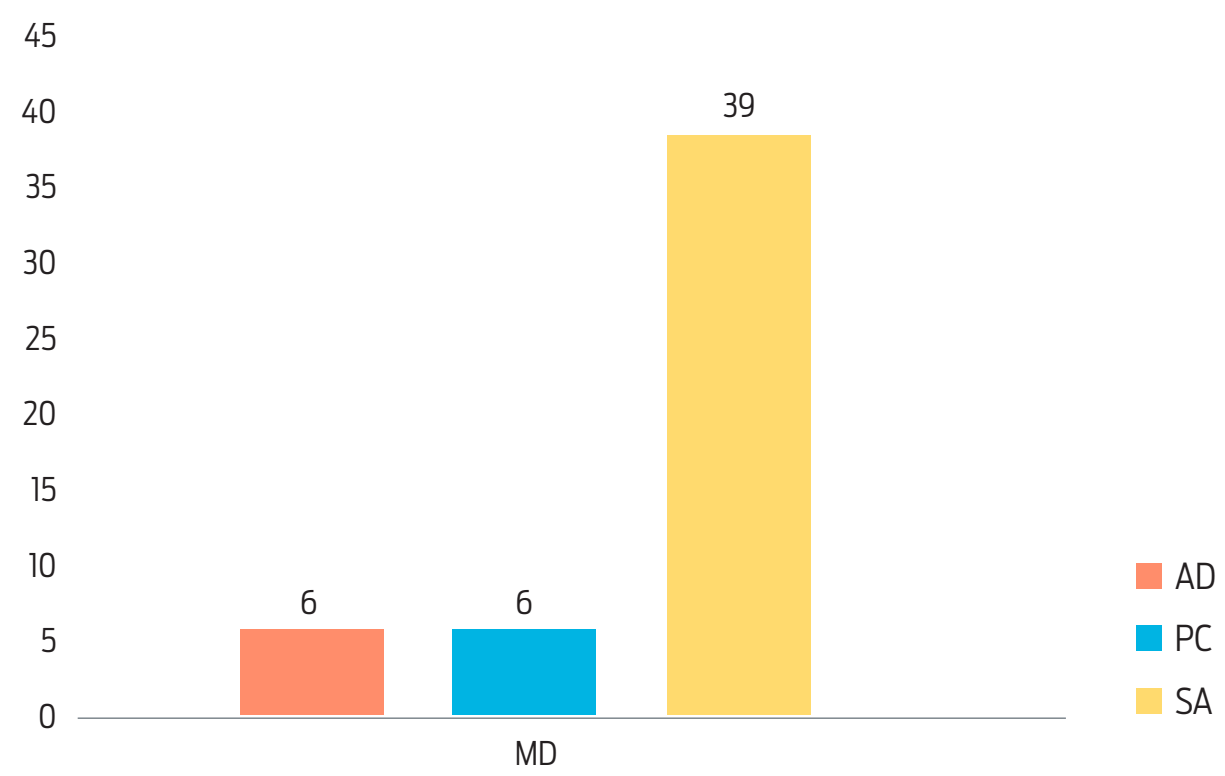

Figura 6. Distribución de miositis dorsal en pacientes que recibieron TOCE. MD miositis dorsal. AD adiestramiento, PC prueba completa, SA salto.

las lesiones tendinosas y ligamentosas de las que la desmitis del aspecto proximal del ligamento suspensor o tercer músculo interóseo, es la más común. ${ }^{1,2}$

La segunda patología con mayor incidencia fue miositis dorsal con $15 \%$ y 51 terapias aplicadas, de las que, al igual que la DPLS, los pacientes fueron caballos de salto, adiestramiento y prueba completa. Los problemas de espalda en caballo son primarios o secundarios y representan una de las primeras causas de alteración en el paso y el rendimiento. La identificación de alteraciones en la espalda son difíciles en el caballo, lo que representa un reto para el clínico (Figura 6). ${ }^{3}$

La siguiente lesión con mayor incidencia en el estudio fue exostosis anillada con 33 aplicaciones a caballos dedicados a salto, adiestramiento y paseo (con lesiones exclusivamente en miembros torácicos). Exostosis anillada es el nombre que recibe la periostitis con hueso de neoformación en la región de la cuartilla. Se clasifica en articular o no articular si el proceso involucra la articulación interfalán-gica proximal o distal, por lo que a la osteoartritis de la articulación interfalángica proximal se le conoce como exostosis anillada alta (Figura 7). ${ }^{4}$

La desmitis de la rama lateral del ligamento suspensor representó el $7.43 \%$ del total de terapias aplicadas. La distribución se muestra en la Figura 8. La desmitis de las ramas medial y lateral del ligamento suspensor tienen igual incidencia en miembros anteriores y posteriores de la mayoría de los caballos, aunque es común que solo una rama esté afectada en un miembro. ${ }^{5}$

Por otra parte, la desmitis de ambas ramas del ligamento suspensor representó el $6.5 \%$ de terapias aplicadas (Figura 9).

Las anteriores cinco patologías representaron el $71.51 \%$ del total de aplicaciones, el $28.49 \%$ restante se muestra en el Cuadro 1. 


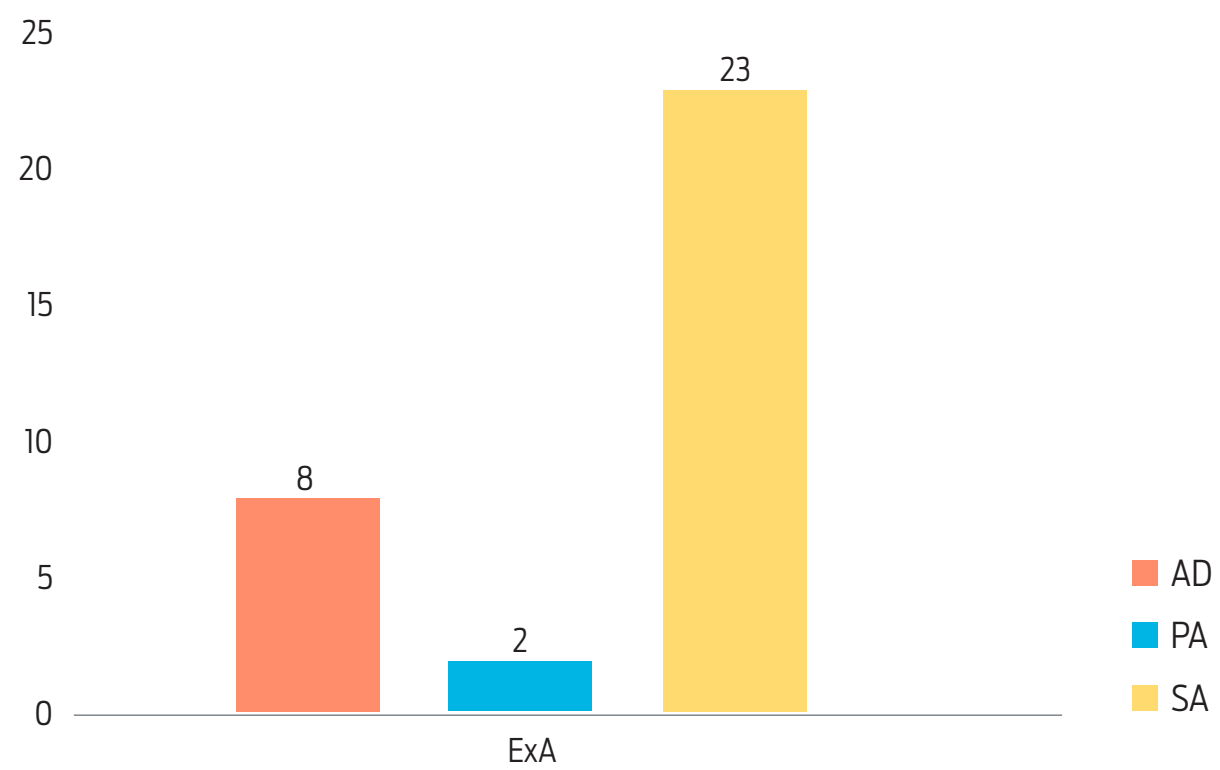

Figura 7. Distribución de exostosis anillada en pacientes que recibieron TOCE. ExA exostosis anillada. AD adiestramiento, PA paseo, SA salto.

\section{DRLLS}

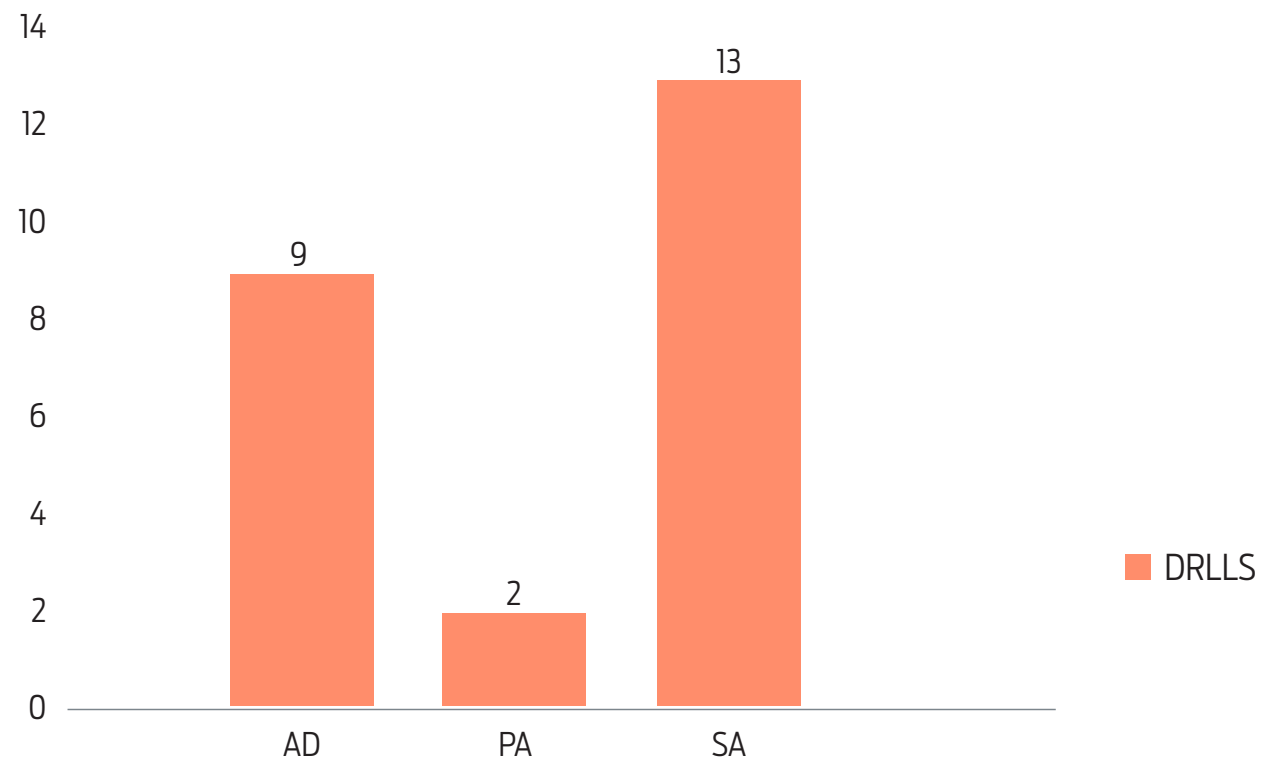

Figura 8. Distribución de desmitis de la rama lateral del ligamento suspensor. AD adiestramiento, PA paseo, SA salto. 


\section{DRsLS}

18
16
14
12
10
8
6
4
2
0

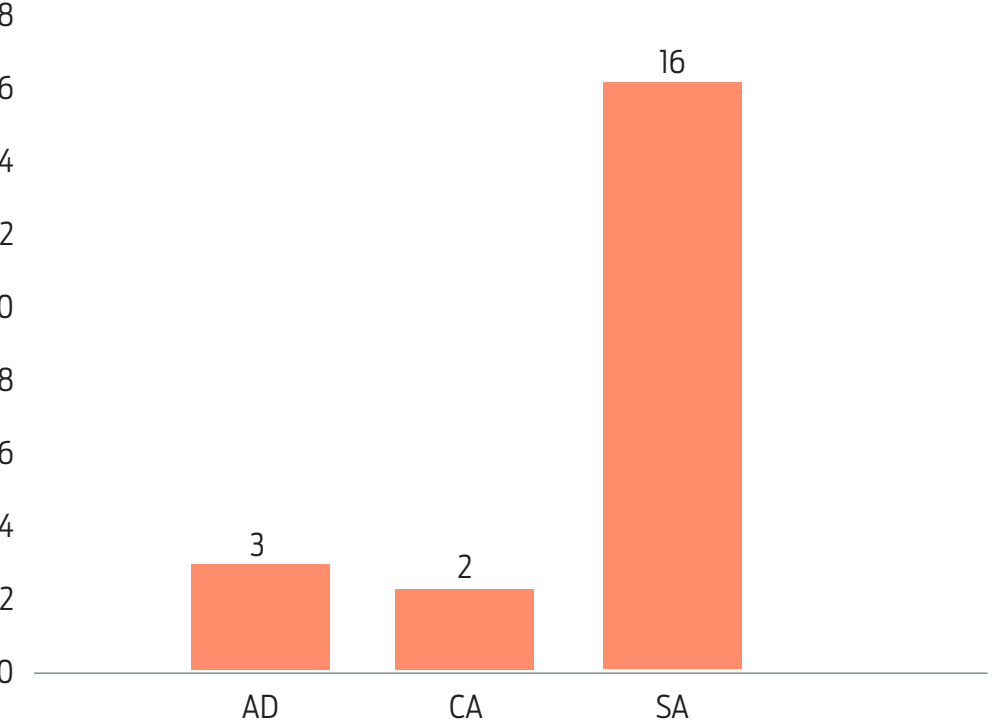

DRsLS

Figura 9. Distribución de desmitis de ambas ramas del ligamento suspensor. AD adiestramiento, CA carreras, SA salto.

Cuadro 1. Terapias aplicadas por cada patología tratada

\begin{tabular}{|c|c|c|c|c|c|}
\hline Patologla & Adiestramiento & Resistencia & Paseo & Salto & Total \\
\hline BB & 1 & & & & 1 \\
\hline CMA & & 8 & & & 2 \\
\hline DCLS & & & & 10 & 10 \\
\hline DLCM & & & & 7 & 7 \\
\hline DLFI & 2 & & & 7 & 9 \\
\hline DLRP & & & & 2 & 2 \\
\hline DLS & & & & 9 & 9 \\
\hline DLsCs & & & & 3 & 3 \\
\hline DRMLS & 3 & & & & 3 \\
\hline ESPO & 2 & & & 5 & 7 \\
\hline FAP & 5 & & & & 5 \\
\hline OCL & & & & 1 & 1 \\
\hline S & & & & 2 & 2 \\
\hline SD & & & & 2 & 2 \\
\hline SN & & & & 4 & 4 \\
\hline SP IV MC & & & & 1 & 1 \\
\hline STC & & & & 1 & 1 \\
\hline TFDP & 3 & & & 4 & 7 \\
\hline TDFS & 2 & & 2 & 6 & 10 \\
\hline TOTAL & 18 & 8 & 2 & 64 & 92 \\
\hline
\end{tabular}


Cuadro 2. Características del equipo de TOCE

\begin{tabular}{l|c|c|}
\multicolumn{1}{l|}{ Caracteristicas } & TOCER \\
\hline Energía & $0.01-0.55 \mathrm{mj} / \mathrm{mm}^{2}$ & $1-5 \mathrm{bar}$ \\
\hline Profundidad terapéutica & $0-125 \mathrm{~mm}$ & $0-30 \mathrm{~mm}$ \\
\hline Frecuencia & $1-8 \mathrm{~Hz}$ & $0.5-21 \mathrm{~Hz}$ \\
\hline & & \\
\hline
\end{tabular}

\section{Tratamiento y evolución}

Para el tratamiento de los pacientes se utilizó el equipo Duolith Medical de Storz Medical, que tiene la capacidad para el tratamiento mediante terapia de onda de choque focal (TOCEF) y terapia de onda de presión radial (TOCER); sus características principales se muestran en el Cuadro 2.

Se aplicaron 273 terapias mediante TOCEF y 50 mediante TOCER. La proporción se ejemplifica en la Figura 10.

La TOCEF se utilizó para el tratamiento de patologías en miembros torácicos y pélvicos, mientras que la TOCER se prefirió para el tratamiento de miositis dorsal únicamente. La diferencia y preferencia por cada tipo de TOCE radica en factores como el tipo de lesión, el criterio de cada clínico, por ejemplo, la TOCER se prefiere en el tratamiento de miositis dorsal, ya que la presión de la onda de choque no disminuye en el tejido muscular ${ }^{6}$ y no se requiere un punto específico para el tratamiento a diferencia de la TOCEF, que tiene mayor capacidad de penetración y es capaz de enfocar la energía en un punto específico, preferida para el tratamiento en inserciones ligamentosas, desmitis, tendinitis y osteoartritis. ${ }^{7}$

\section{Discusión y relevancia clínica}

La onda de choque es generada mediante una liberación súbita de energía química, física o nuclear, la cual se caracteriza por un aumento en la amplitud en un período corto de tiempo, seguida de una onda negativa. ${ }^{8}$ Puede ser generada a través de tres mecanismos: electrohidráulico, piezoeléctrico y electromagnético. El equipo utilizado en el hospital para équidos genera ondas de choque mediante el método electromagnético el cual utiliza una bobina, una membrana y un lente que focaliza la onda. ${ }^{9}$ La onda de presión radial o mal llamada terapia de ondas de choque radial, es generada a través de la aceleración de un proyectil mediante aire comprimido. ${ }^{9}$ La terapia de onda de choque fue utilizada por primera vez en humanos para el tratamiento de cálculos renales ${ }^{10}$. Posteriormente se utilizó en el tratamiento de tejido musculo esquelético y su primer uso en equinos fue reportado en el tratamiento de desmitis proximal del ligamento suspensor. ${ }^{11}$ La terapia de onda de choque actualmente es utilizada para el tratamiento de osteoartritis, tendinitis, desmitis, 


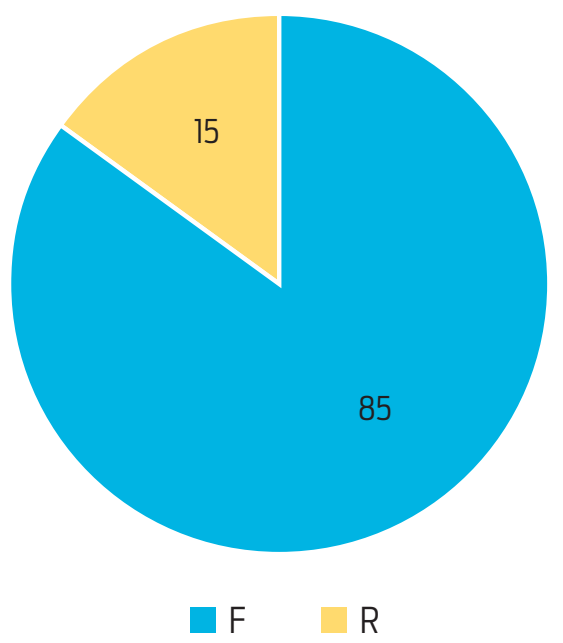

Figura 10. Proporción TOCEF-TOCER.

síndrome navicular en equinos y sus aplicaciones biomédicas siguen en expansión e investigación. ${ }^{2,12}$

La principal patología tratada mediante TOCE en el Hospital para Équidos de la FMVZ-UNAM durante el período 2011-2015, fue la desmitis proximal o del origen del ligamento suspensor del menudillo, con un total de 102 aplicaciones, la cual representa el $31.57 \%$ de las 323 terapias aplicadas, seguida de miositis dorsal con un $15.78 \%$ y exostosis anillada con el $10.21 \%$. Sin embargo, aunque la TOCE representa una opción no invasiva y eficaz para el tratamiento de patologías como desmitis, tendinitis, osteoartritis, síndrome navicular, miositis ${ }^{12}$ y a pesar de que las patologías son comunes en las disciplinas ecuestres de los caballos de estudio, el mayor porcentaje de pacientes que son remitidos para TOCE son de salto y adiestramiento. Patología que mediante múltiples estudios han demostrado responder de forma favorable al tratamiento mediante TOCE. ${ }^{11}$ Por otra parte, la TOCE representó el 17.9\% del total de casos clínicos durante el período de estudio, sin embargo, no es posible compararlo con las diferentes causas de remisión en el HE-FMVZ-UNAM por falta de estudios de caso al respecto.

\section{Agradecimientos}

Se agradece a la mvz María Fernanda Garcés Loza por la recopilación de los datos mediante los expedientes físicos del HE.

\section{Conflictos de interés}

No existe algún motivo de conflictos de interés, ya que el estudio únicamente tiene el objetivo de conocer las principales aplicaciones de la TOCE y las características de los pacientes que fueron remitidos para dicha terapia en el hospital para équidos de la FMVZ-UNAM durante 2011-2015.

\section{Contribución de los autores}

Missael García Márquez: Edición, redacción y análisis de contenido 
Carlos Antonio López Díaz: Asesoría en estadística descriptiva

Alejandro Rodríguez Monterde: Líder de equipo de redacción y edición de contenido. Análisis clínico y corrección .

\section{Referencias}

1. Dyson S. Proximal suspensory desmitis in the sports horse in forelimbs. American Association of Equine Practitioners. 2007:93 -8. Disponible en: https:// pdfs.semanticscholar.org/74f3/00120869fdba7241c529b41799a5529a $18 f 9$. pdf. Citado diciembre 2019.

2. Dyson S. Proximal suspensory desmitis in the forelimb and the hindlimb. AAEP Proc. 2000;46:137-42. Disponible en: https://pdfs.semanticscholar.org/92bf/633d51377a8bfba6fd3b5906575dae333812.pdf. Citado diciembre 2019.

3. García-López JM. Neck, back, and pelvic pain in sport horses. Veterinary Clinics of North America - Equine Practice. 2018;Aug;34(2):235-251.

4. García Márquez M, Rodríguez Monterde A. López-Díaz C. Casos clínicos referidos de 2011-2015 para terapia de ondas de choque extracorporales (TOCE), al hospital del departamento de Medicina, Cirugía y Zootecnia para Équidos, de la FMVZ-UNAM: estudio retrospectivo. Universidad Nacional Autónoma de México; 2018.

5. Dyson SJ, Arthur RM, Palmer SE, Richardson D. Suspensory ligament desmitis. Vol. 11, The veterinary clinics of North America. Equine practice. 1995. p. 177-215.

6. Schmitz C, Császár NBM, Milz S, Schieker M, Maffulli N, Rompe JD, et al. Efficacy and safety of extracorporeal shock wave therapy for orthopedic conditions: a systematic review on studies listed in the PEDro database. $\mathrm{Br}$ Med Bull. 2015;116(1):115-38. Disponible en: https://www.ncbi.nlm.nih.gov/pubmed/26585999. Citado diciembre 2019.

7. McClure S, Maier M, Schmitz C. Extracorporeal shock wave therapy [internet]. En: Current therapy in equine medicine. 5a ed: USA, Elsevier; 2003. p. 562-6. http://dx.doi.org/10.1016/B978-0-7216-9540-2.50168-7. Citado diciembre 2019.

8. Mcclure S, Dorfmu C, Phys D. Extracorporeal shock wave therapy: theory and equipment. 2004;2(4):348-57.

9. Scott R. McClure. Extracorporeal shock wave therapy in horses: what we know [internet]. AAEP; 2016. Disponible en: https://aaep.org/horsehealth/extracorporeal-shock-wave-therapy-horses-what-we-know. Citado diciembre 2019.

10. Chaussy C, Brendel W, Schmiedt E. Extracorporeally induced destruction of kidney stones by shock waves [internet]. Lancet; 1980 (Dec 13);316(8207):1265-8. Disponible en: https://www.sciencedirect.com/science/article/pii/S01406736 80923351?via\%3Dihub. Citado: el 23 de agosto de 2019.

11. Boening KJ, Lo S, Weitkamp K, Matuschek S. Radial extracorporeal shock wave therapy for chronic insertion desmopathy of the proximal suspensory ligament. AAEP Proc. 2000;46:203-7. Disponible en: http://www.ivis.org/proceedings/ aaep/2000/203.pdf?origin=publication_detail. Citado diciembre de 2019.

12. Mcclure S, Weinberger T, Pferde FTA. Extracorporeal shock wave therapy: clinical applications and regulation. 2004;2(4):358-67. Disponible en: https:// www.sciencedirect.com/science/article/abs/pii/S1534751604000125. Citado diciembre de 2019 